\title{
Rare hemi-retinal phenotype in a cone-rod dystrophy demonstrated by optomap ultra-widefield imaging
}

\author{
Ala Noaman ${ }^{1}$, V. Swetha E. Jeganathan ${ }^{1,2 *}$ and Andrew Blaikie ${ }^{3}$ \\ ${ }^{1}$ University of Edinburgh, Scotland \\ ${ }^{2}$ Department of Ophthalmology, University Hospital Ayr, Scotland \\ ${ }^{3}$ Department of Ophthalmology, NHS Fife, Scotland
}

\section{Case report}

Our patient first presented at age 7 , with best-corrected visual acuity $6 / 9$ in both eyes. Her disease relentlessly progressed resulting in blindness registration with acuities of $6 / 60$ bilaterally. She also lost colour vision and had photophobia. She had no known family history of visually impairing eye conditions.

Fundoscopy elicited bulls-eye maculopathy and segmental bone spicule pigmentation. Ultra-wide-field retinal imaging (Optomap) revealed hemiretinal disease affecting the superior retina (Figures $1 \mathrm{a}$ and $1 \mathrm{~b}$ ). Goldmannperimetry demonstrated bilateral large paracentral scotomas involving the inferior portions of her vision with unusual conformance to the horizontal meridian (Figures $2 a$ and $2 b$ ). A diagnosis of CRD was made as electroretinography showed reduced amplitudes (cone > rod) consistent with cone and rod dysfunction. Genetic testing revealed a mutation of $\mathrm{C} 1490 \mathrm{Y}$ in the ABCA4 gene.

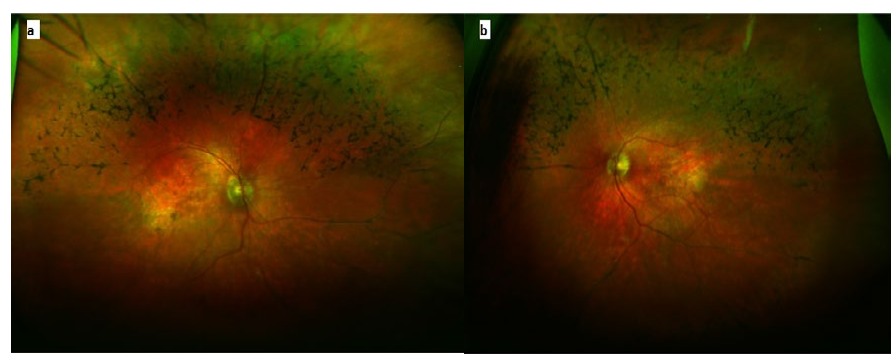

Figure 1: Optomap ultra-wide-field retinal imaging of both eyes. Bone-spicule pigmentation affecting the superior half of the retina is seen in the (a) left eye and (b) right eye.

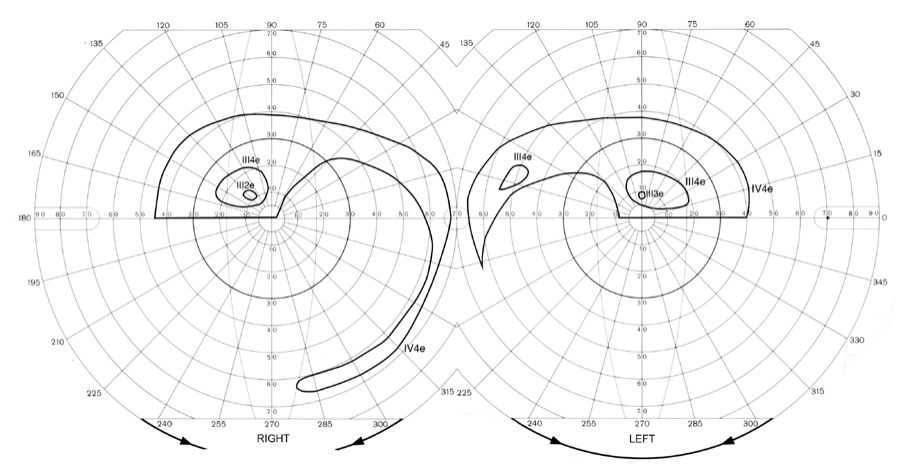

Figure 2. Goldmann visual fields with bilateral constricted fields with pseudo--altitudinal defects seen in the (a) left eye and (b) right eye.

\section{Discussion}

ABCA4 gene mutations can lead to a spectrum of retinal degenerations ranging from Stargardt disease to CRD and $\sim 600$ variations exist depending on residual protein activity. Our patient presented with visual fields and fundal signs not typical of CRD which typically affects the whole fundus with minimal clinical signs and rarely 'perivenular bone spicule pigmentation'. Furthermore, the visual field defects have been difficult to class as 'pseudo' or 'genuine'.

The literature describes pseudo-altitudinal field defects in conerod dystrophy in autosomal recessive forms and chromosome $19 q$ mutations, although these are relatively rare presentations $[1,2]$. Moreover, retinal causes of altitudinal defects are rare. Zeiss et al described X-linked retinal atrophy in canines with focal retinal loss due to lyonisation[3].Lyonisation has only been described in X-linked ocular albinism, $\mathrm{X}$-linked retinoschisis, $\mathrm{X}$-linked retinitis pigmentosa and choroideremia in humans $[4,5]$. This raises the possibility that the mutation identified (ABCA4 missense mutation) is incidental to the clinical findings and in fact this patient has an unrecognised X-linked retinal dystrophy with lyonisation affecting only one half of the fundus, similar to that of sectoral retinitis pigmentosa. Optomap technology was useful in providing wide-field views of the retina and in showing the phenotype of the disease.

\section{Acknowledgment}

Poster presentation at Scottish Ophthalmological Club Meeting, Stirling, $21^{\text {st }}$ February 2014.

Correspondence to: Dr. V. Swetha E. Jeganathan, Department of Ophthalmology, University Hospital Ayr, Dalmellington Road, Ayr KA6 6DX, Scotland, E-mail: vswetha@ausdoctors.net

We report an unusual hemi-retinal phenotype in cone-rod dystrophy (CRD) diagnosed with ultrawide- field retinal imaging. To date, a hemiretinal phenotype has not been described in CRD.

Key words: cone-rod dystrophy, ACA4 (ABCR) gene, autosomal recessive, hemiretinal phenotype, lyonisation

Received: November 10, 2015; Accepted: December 02, 2015; Published: December 07, 2015 


\section{References}

1. Heckenlively JR (1987) RP cone-rod degeneration.Trans Am OphthalmolSoc85: 43870. [Crossref]

2. Evans K, Duvall-Young J, Fitzke FW, Arden GB, Bhattacharya SS, et al. (1995) Chromosome 19q cone-rod retinal dystrophy. Ocular phenotype. Arch Ophthalmol113: 195-201.[Crossref]

3. Zeiss CJ, Acland GM, Aguirre GD (1999) Retinal pathology of canine x-linked progressive retinal atrophy, the locus homologue of RP3. Invest Ophthalmol Vis Sci 40: 3292-3304.[Crossref]

4. Cherfan CG, Link TP, Babovic-Vuksanovic D, Ellison JW, Brodsky MC (2013) Retina mosaicism in a girl with an X-Y translocation. Br J Ophthalmol 97: 243.[Crossref]

5. Wuthisiri W, Lingao MD, Capasso JE, Levin AV (2013) Lyonization in ophthalmology CurrOpinOphthalmol 24: 389-97.[Crossref]

Copyright: (C2015 Noaman A. This is an open-access article distributed under the terms of the Creative Commons Attribution License, which permits unrestricted use, distribution, and reproduction in any medium, provided the original author and source are credited. 